\title{
Atom Probe Tomography of Small-Molecule Organic Materials
}

Jeramy Zimmerman ${ }^{1}$, Andrew Proudian ${ }^{2}$, Matthew Jaskot ${ }^{3}$, Paul Niyonkuru ${ }^{4}$, Roland Bennett ${ }^{5}$, Jacob Bingham ${ }^{6}$ and Shubham Vyas ${ }^{6}$

${ }^{1}$ Colorado School of Mines, Physics, GOLDEN, Colorado, United States, ${ }^{2}$ Colorado School of Mines, Physics, Golden, Colorado, United States, ${ }^{3}$ Colorado School of Mines, GOLDEN, Colorado, United States, ${ }^{4}$ Colorado School of Mines, Golden, Colorado, United States, ${ }^{5}$ Colorado School of Mines, GOLDEN, Colorado, United States, ${ }^{6}$ Colorado School of Mines, United States

Organic small molecules are the backbone of various electronic devices, including organic photovoltaics (OPVs) and organic light-emitting diodes (OLEDs), and appear in biological materials. Many organic electronic devices contain blends of two or more molecules where the phase separation and interfacial properties are crucial to device operation. Conventional $\mathrm{nm}$-scale imaging techniques, such as transmission electron microscopy (TEM), are challenging to perform without significantly damaging the material and often provide limited information in these materials. However, atom probe tomography (APT) provides previously inaccessible information and enables evaluation of nanometer-scale morphology with local mass spectral labeling, enabling impurity analysis, morphological development, and understanding of degradation pathways in molecular organic electronic materials blends.

Sample specimen preparation is critical for generating high-quality APT data results. These molecules used in organic electronics are stable on vacuum thermal evaporation (VTE), enabling us to evaporate 50 to $100 \mathrm{~nm}$-thick films onto smooth pre-formed silicon tips with a radius of curvature of 150 to $500 \mathrm{~nm}$. At these large radii, we can analyze the materials under relatively typical APT conditions of temperatures of 20-40 K, a laser power of 1-20 pJ, and voltage of 5000 to $9000 \mathrm{~V}$. These analysis conditions are possible for these very large radii because the molecules have small evaporation fields (typically $2-10 \mathrm{~V} / \mathrm{nm}$ ).

Using these conditions, we have found that most evaporable molecules of interest in organic electronics can be analyzed, ranging from fullerenes (e.g., $\mathrm{C}_{60}$ ) to conjugated systems (e.g., 3,3'-Bis(carbazol-9-yl)biphenyl) to chelated metalorganics (e.g., tris(2-phenylpyridine)iridium or $\left.\operatorname{Ir}(\mathrm{ppy})_{3}\right)$ without indication of fragmentation. The lack of fragmentation owes itself to the relatively weak inter-molecular Van der Waals forces holding molecules into a solid, which are significantly smaller than the intramolecular chemical bonds within each molecule. In these systems, sub-nanometer spatial resolution, sub-dalton mass discrimination, and the ability to identify impurities or degradation products below 0.01 atomic\% have been achieved $[1,2,3]$.

Although most thermally evaporable small molecules we have analyzed using APT cleanly field evaporate as entire molecules, we have recently discovered a molecule, BSB (4,4'-Di(triphenylsilyl)-biphenyl), where the molecular dication (i.e., the $2+$ state) undergoes post-ionization fragmentation mid-flight (Figure 1). The molecular cleavage occurs at a silicon-phenyl bond, resulting in fragment peaks in the mass spectrum and dissociation tracks in a double-hit correlation histogram.[4] We use density functional theory (DFT) to evaluate the energetics of the process to show that dications states of many molecules are energetically stable, but it is energetically downhill for a fully relaxed BSB dication to fragment into two singly ionized molecular ions (Figure 2). We will present DFT and APT results of several molecules with these Si-phenyl bonds where molecular fragmentation is and is not observed and relate these results to molecular properties. 


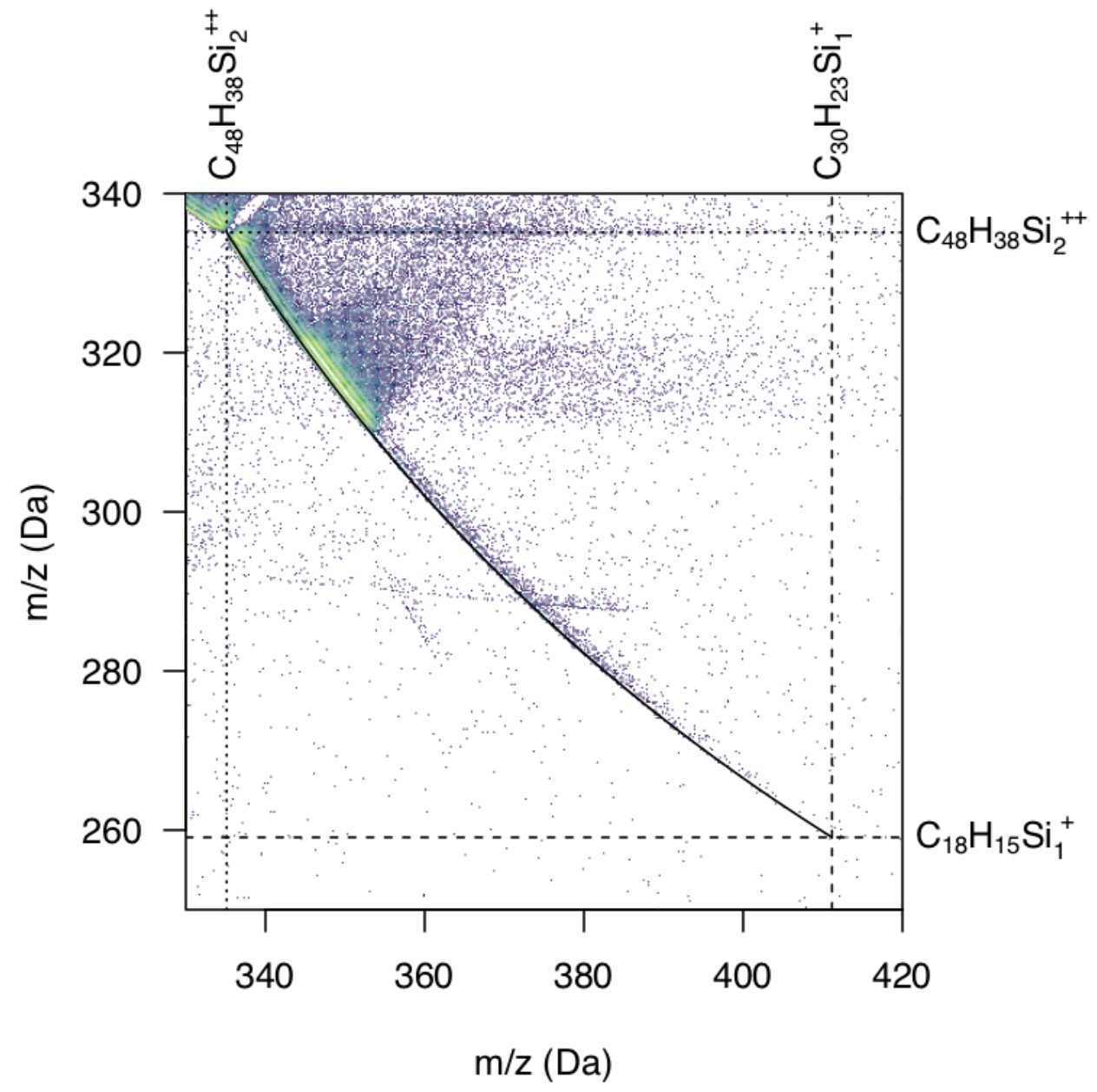

Figure 1. Correlation histogram of BSB showing post-ionization fragmentation of the doubly ionized molecule into two fragments.

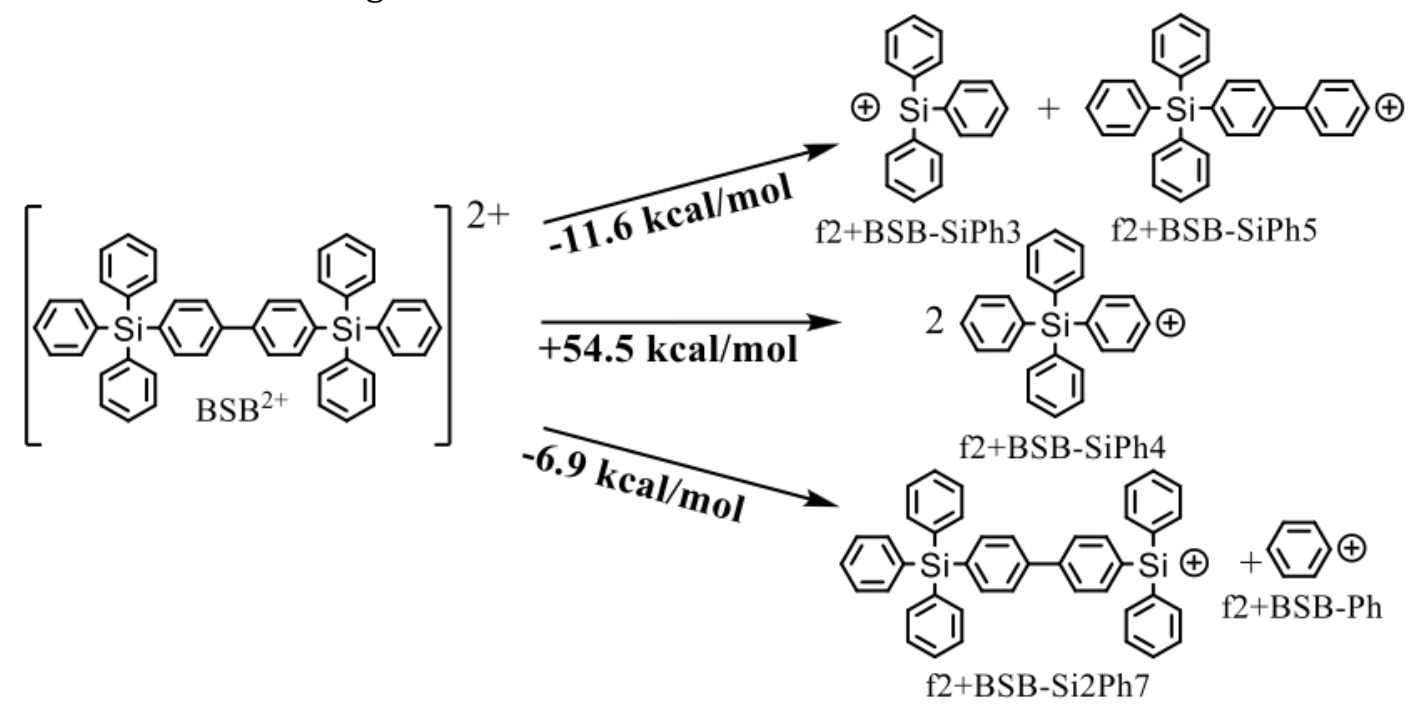

Figure 2. Molecular structure of BSB and daughter fragments and the energy difference of each process showing two fragmentation pathways. References 
[1] A.P. Proudian, M.B. Jaskot, C. Lyiza, D. R. Diercks, B.P. Gorman, and J.D. Zimmerman, Effect of Diels-Alder Reaction in C60-Tetracene Photovoltaic Devices, NanoLetters 16, 6086-6091 (2016)

[2] A.P. Proudian, M.B. Jaskot, D.R. Diercks, B.P. Gorman, J.D. Zimmerman, Atom Probe Tomography of Molecular Organic Materials: Sub-Dalton Nanometer-Scale Quantification, Chemistry of Materials 31, 2241-2247 (2019).

[3] Data analyzed in this presentation is performed using our R for Atom Probe Tomography (rapt) analysis package: A.P. Proudian, rapt: R for atom probe tomography, https://github.com/aproudian2/rapt. (2020)

[4] Saxey, D. W. Correlated ion analysis and the interpretation of atom probe mass spectra. Ultramicroscopy 111, 473-479 (2011)

[5] This work was supported in part by grant DE-SC0018021 funded by the U.S. Department of Energy, Office of Science Early Career program. 